\title{
Craniotabès chez un nouveau-né
}

\author{
Yuko Wada MD, Katsuyuki Kubo MD, Shinichi Tsubata MD
}

Citation : CMAJ 2020 October 5;192:E1163. doi : 10.1503/cmaj.200192-f

Voir la version anglaise de l'article ici : www.cmaj.ca/lookup/doi/10.1503/cmaj.200192

$\mathbf{U}$ ne femme de 40 ans qui était G2P2 a accouché d'un garçon à terme après une grossesse sans complications. À l'examen physique du nouveau-né, nous n'avons pas pu palper les os crâniens sur le dessus de sa tête. Ses membres et son visage ne présentaient pas d'anomalies laissant supposer une malformation syndromique. La tomodensitométrie crânienne a montré des zones importantes de retard d'ossification le long de la suture sagittale, et plusieurs petites lésions aux os temporal et occipital, que nous n'avions pas détectées à l'examen physique (figure $1 \mathrm{~A}$ et $\mathrm{B}$ ).

Les tests en laboratoire effectués au $5^{\mathrm{e}}$ jour de vie de l'enfant ont donné les résultats suivants : taux sérique de 25-hydroxyvitamine $D$ de $14,1 \mathrm{ng} / \mathrm{mL}$ (plage normale $20-100 \mathrm{ng} / \mathrm{mL}$ ); et taux de parathormone accru de $91 \mathrm{pg} / \mathrm{mL}$ (plage normale $10-60 \mathrm{pg} / \mathrm{mL}$ ). Nous avons diagnostiqué une carence en vitamine $\mathrm{D}$ et une hyperparathyroïdie secondaire. Les radiographies des membres n'ont pas montré de signes de rachitisme. La mère n'a aucun antécédent de syphilis et n'a pas restreint son alimentation ou son exposition au soleil. Cependant, son taux sérique de 25 -hydroxyvitamine $D$ était bas $(9,4 \mathrm{ng} / \mathrm{mL}$ [plage normale $30-100 \mathrm{ng} / \mathrm{mL}$ ]). Nous avons prescrit une alimentation normale avec une supplémentation en vitamine $D$, qui a permis d'améliorer les taux sériques de vitamine $D$. Les os crâniens du nourrisson se sont solidifiés à 2 mois, et il s'est développé normalement par la suite.

L'incidence de craniotabès, défini comme un ramollissement des os du crâne, varie entre $20 \%$ et $30 \%$ dans le monde (bien que les cas graves comme celui que nous décrivons soient rares) et est en corrélation directe avec le taux de vitamine $D$ de la mère ${ }^{1}$. Avec une nutrition adéquate, il se résout spontanément en 2-3 mois ${ }^{1}$. Le craniotabès est aussi associé à des pathologies comme le rachitisme, la syphilis congénitale ou les dysplasies squelettiques ${ }^{1}$. Notre cas était vraisemblablement causé par une carence maternelle en vitamine $D$. La prévalence de cette carence chez les femmes enceintes varie de $50 \%$ à $70 \%$ dans les pays développés, en fonction de la région ${ }^{2}$.
L'American College of Obstetricians and Gynecologists recommande que les femmes enceintes prennent $600 \mathrm{UI}$ de suppléments de vitamine D par jour; toutefois, 1000-2000 UI/jour pourraient être nécessaires s'il y a une carence ${ }^{3}$.

\section{Références}

1. Yorifuji J, Yorifuji T, Tachibana K, et al. Craniotabes in normal newborns: the earliest sign of subclinical vitamin D deficiency. J Clin Endocrinol Metab. 2008;3:1784-8.

2. Saraf R, Morton SM, Camargo CA Jr, et al. Global summary of maternal and newborn vitamin D status - a systematic review. Matern Child Nutr 2016;12:647-68.

3. ACOG Committee on Obstetric Practice. ACOG Committee Opinion No. 495: Vitamin D: screening and supplementation during pregnancy. Obstet Gynecol 2011;118:197-8 [reconduit en 2017].

Intérêts concurrents : Aucun déclaré.

Cet article a été révisé par des pairs.

Les auteurs ont obtenu le consentement des parents.

Affiliation : Département de pédiatrie, Hôpital de la Croix-Rouge de Toyama, Toyama, Japon

Remerciements : Les auteurs remercient le Dr Neil Bowles pour son aide à la révision du manuscrit.

Correspondance : Yuko Wada, ykwada22@gmail.com 